\title{
CONGENITAL VERTICAL TALUS
}

\author{
G. C. Lloyd-Roberts and A. J. Spence, London, England
}

From the Orthopaedic Department, the Hospital for Sick Children, Great Ormond Street, London, and the Institute of Orthopaedics and Royal National Orthopaedic Hospital, London and Stanmore

Congenital vertical talus is an uncommon but well known cause of severe flat foot in children. It is characterised by distinct clinical and radiological features which are, however, sometimes simulated in cases of flat foot from other causes, known or unknown. Our interest was aroused when we found difficulty in distinguishing between this truly congenital abnormality and severe flat foot with talo-navicular subluxation. It seemed desirable therefore to review our cases critically and to establish criteria upon which we could differentiate the one from the other, preparatory to a study of the natural history of the anomaly and the methods of treatment available. In this communication we are concerned almost exclusively with the diagnosis and differential diagnosis of congenital vertical talus-other observations will be incidental.

Although there are numerous continental papers on congenital vertical talus there are few in English. In most, emphasis has been on treatment rather than on the clinical aspects. More recently however, in discussing treatment, Hark (1950) and Osmond-Clarke (1956) have mentioned some of the clinical features. The literature up to 1939 was well reviewed by Lamy and Weissman (1939).

\section{CLINICAL MATERIAL}

We have classified thirty-two feet in twenty-two patients as examples of congenital vertical talus. We offer no apology for reporting so small a number, for we find ourselves in company with others who have been critical in accepting this diagnosis. Osmond-Clarke (1956) reported an incidence of one in 121 in a special clinic for congenital foot deformities. Our patients have been traced by reference to the diagnostic indices of the Hospital for Sick Children (seventeen) and the Royal National Orthopaedic Hospital (five)-covering eighteen and twenty-seven years respectively. Their ages are between infancy and fifteen. Few of the severe flat feet seen at the Hospital for Sick Children are radiographed, and it is probable that some cases of vertical talus were classified under other diagnoses. The facts that have led to our conclusions about diagnostic criteria and differential diagnosis have come not only from these patients but also from a study of a far greater number of severe flat feet of varying causes.

\section{THE CLINICAL AND RADIOLOGICAL FEATURES OF CONGENITAL VERTICAL TALUS}

The deformity, as its name implies, is present at birth and is usually noticed within the first few weeks. From the first there is a prominence in the sole from which the heel and forefoot rise in a gentle curve (Figs. 1 and 2). The forefoot is dorsiflexed; so a diagnosis of congenital talipes calcaneus is commonly made if the associated equinus deformity of the heel is overlooked (Figs. 1 and 3). Deep creases may be present in front of the ankle (Fig. 3) but eversion of the heel and abduction of the forefoot are not conspicuous in infancy. From the first the deformity is fixed and does not yield to forceful manual pressure. The radiograph is characteristic at a very early age (Fig. 1) for it shows not only the curved outline of the soft tissue of the sole and dorsiflexion of the forefoot, but also a vertical talus and a calcaneum that points into equinus.

When walking begins - this is not apparently delayed-the foot is obviously flat. It is also rigid, so that the foot looks almost the same whether weight bearing or not. The heel

VOL. $40 \mathrm{~B}$, NO. 1, FEBRUARY 1958 


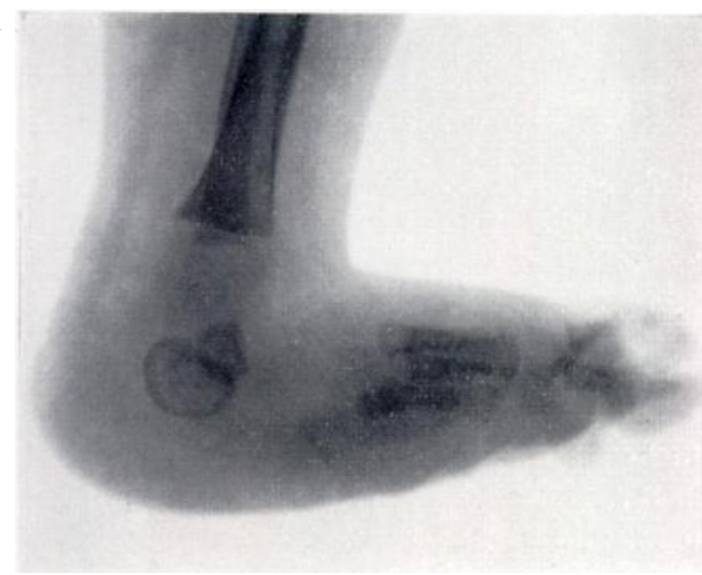

FIG. 1

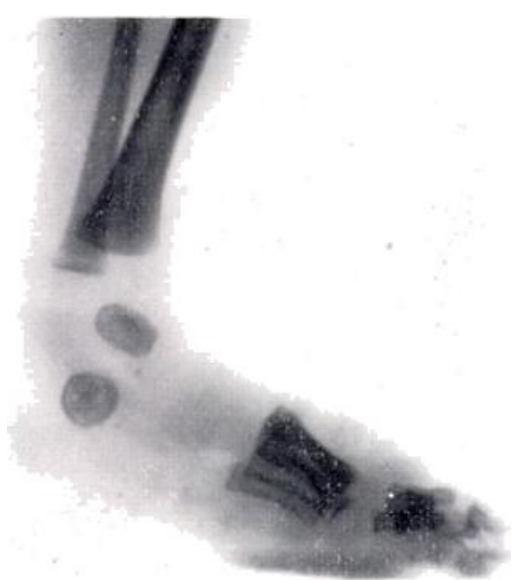

FIG. 2

Figure 1-Congenital vertical talus in an infant of four weeks. Note the perpendicular talus and the equinus calcaneum. The soft-tissue outline of the sole and dorsiflexion of the forefoot are seen. (A normal foot is shown in Figure 2.)

becomes everted and the forefoot becomes abducted on the medially displaced and now readily palpable talus. The heel remains in equinus and the posterior part of the calcaneum fails to touch the ground on standing (Fig. 4). Development of the tarsal bones now permits recognition of some of the specific radiological features (Figs. 5 to 7). The calcaneum is narrower at its anterior end and becomes somewhat beak-shaped. The talus, lying almost in line with the tibial shaft, becomes narrow at the waist and resembles an hour-glass. When the navicular bone shows it appears to be dislocated from the head of the talus and lies in contact with the narrowed body of the bone (Fig. 6). The dislocated navicular bone carries with it the remaining tarsal and metatarsal bones. so that there is dorsal displacement of the foot at the mid-tarsal joint. Elevation of the first metatarsal bone may cause plantar flexion and rigidity of the first metatarsophalangeal joint (Fig. 4). Osteochondritis of the navicular (Fig. 6), with the changes typical of Köhler's disease, sometimes occurs. The navicular finally becomes wedge-shaped towards the sole (Fig. 7). In the antero-posterior radiograph the talus projects widely to the medial side of the calcaneum.

In later childhood the symptoms are an awkward and clumsy gait and distortion of the shoes: fixed valgus deformity of the heel causes rapid distortion of the uppers, and the heels are constantly in need of repair on their inner sides. There may also be symptoms from associated abnormalities, which were present in more than half the patients reported here. Pain appeared for the first time at the age of

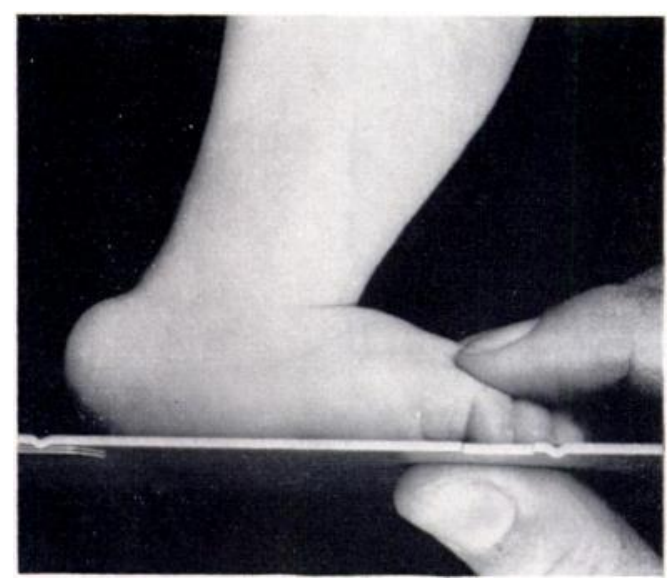

Fig. 3

Appearance of foot at four months. Note the heel in equinus and the forefoot in dorsiflexion. A deep crease is seen in front of the ankle. twelve in one patient and at thirteen in another.

In summary, diagnosis rests upon the shape of the foot, coupled with its rigidity and a characteristic radiograph. If doubt arises on looking at the radiograph the cause of the flat foot is likely to be other than congenital vertical talus. 


\section{CLINICAL TYPES OF CONGENITAL VERTICAL TALUS}

The patients that we have examined fall into four groups. according to the presence or absence of associated defects, and their nature: 1) with arthrogryposis (ten patients); 2) without associated defect-that is, the vertical talus is an isolated congenital defect (nine

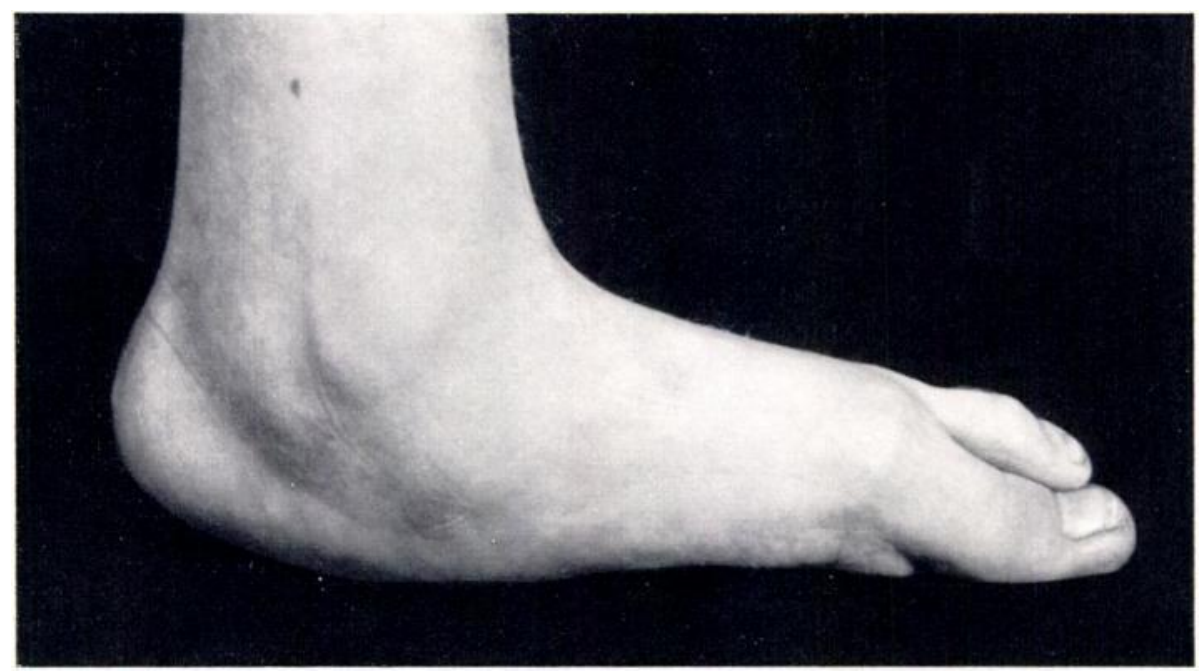

FIG. 4

Congenital vertical talus in a child of six years showing the characteristic boat-shaped foot and the point of the heel off the ground. Note the plantarflexion of the big toe compensating for the elevated first metatarsal head.

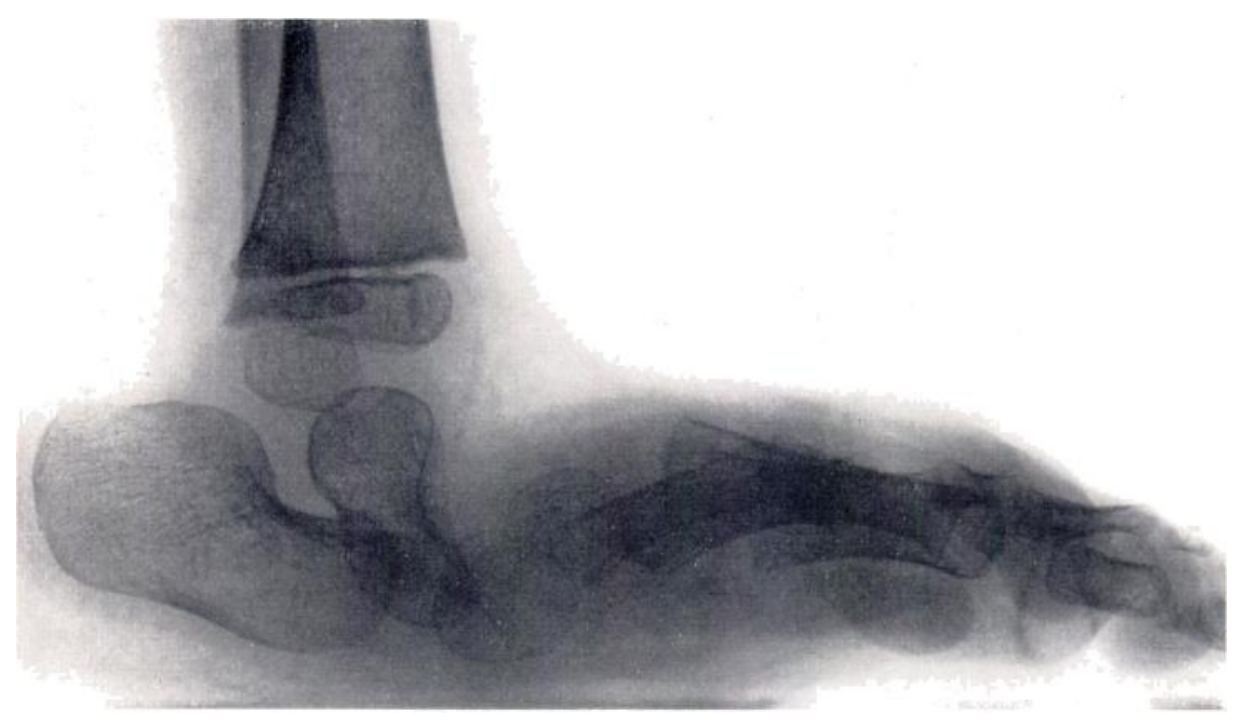

FIG. 5

Aged three years-illustrating the position and shape of the talus, the equinus calcaneum and the dorsiflexed forefoot. The outline of the sole can be seen in this standing radiograph.

patients): 3) with spina bifida (two patients); 4) with neurofibromatosis (one patient). There is no difference between these groups clinically or radiologically, so far as the feet are concerned.

Arthrogryposis is so often present that it is tempting to speculate on its place in the etiology of those cases that apparently exist as single abnormalities or with spina bifida. 
Both types have in common the rigidity and resistance to treatment that characterise manifest arthrogryposis. It is unfortunately not possible to establish the truth or otherwise of the hypothesis that arthrogryposis plays a part.

The deformities associated with arthrogryposis were protean and included five dislocated

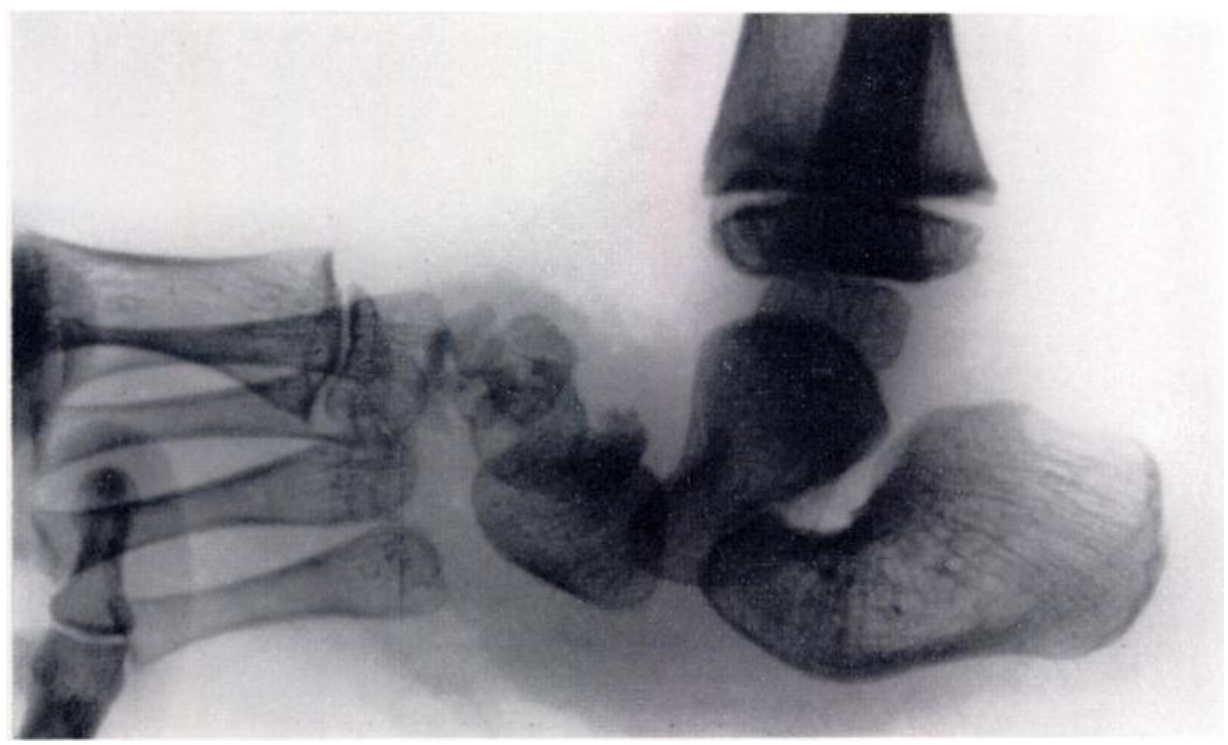

Fig. 6

Aged six years-illustrating the features shown in Figure 5 together with the navicular dislocition and epiphysitis.

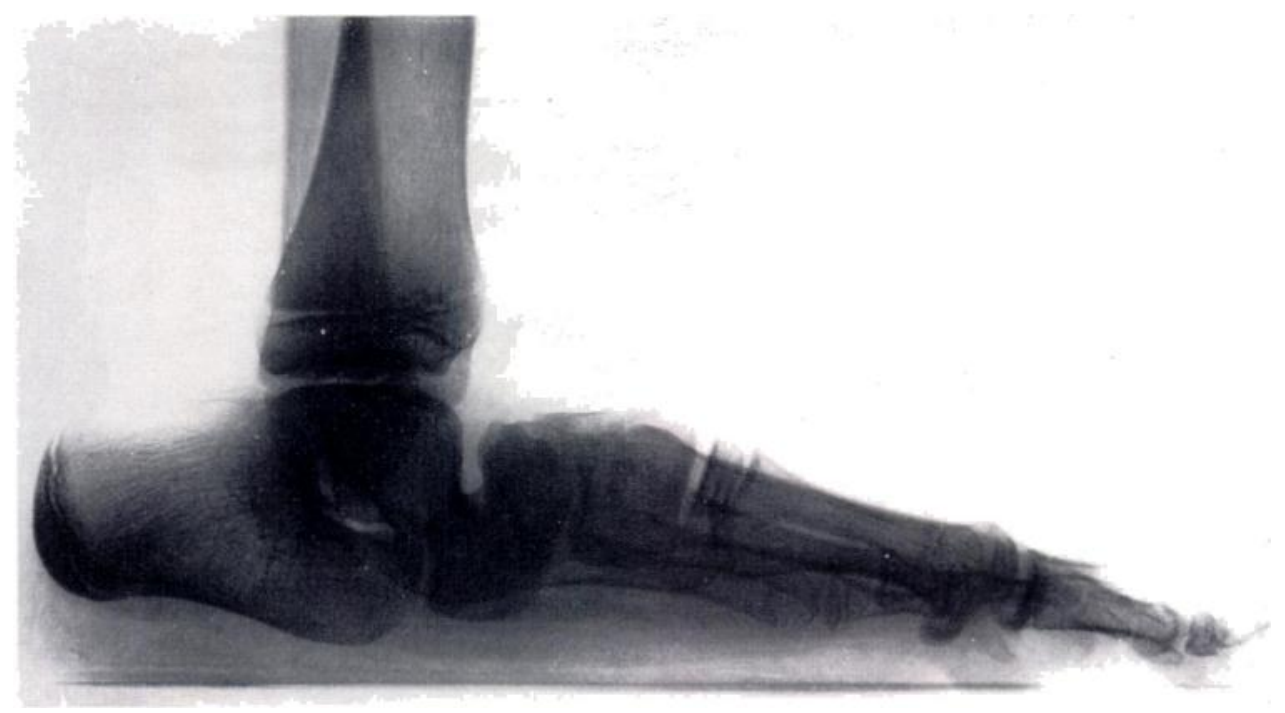

Fig. 7

Aged twelve years-the bones are approaching maturity and retain the features of early childhood in addition to the wedging of the navicular and the beak-shaped calcaneum.

hips. Denis Browne (personal communication) stresses the association between valgus feet of any type in infancy and congenital dislocation of the hip. This observation is supported by our findings in this arthrogrypotic group.

Spina bifida was in one case manifest (with a meningocele) and in the other occult. 
In patients with meningoceles the deformity may be more common than it appears here, because, being often relatively unimportant. it may be overlooked. Figure 1 illustrates the foot of an infant in whom spina bifida occulta was found. The implication is that the deformity is not simply an extreme example of paralytic flat foot; that deformity appears later. has a

TABLE I

incidence or Associated Defects in Twenty-two Patients with Congenital Vertical Talus

\begin{tabular}{|c|c|c|}
\hline Clinical type & Patients & Feet \\
\hline In association with arthrogryposis & 10 & 16 \\
\hline No associated abnormality & 9 & 13 \\
\hline In association with spina bifida & 2 & 2 \\
\hline In association with neurofibromatosis & 1 & 1 \\
\hline Total & 22 & 32 \\
\hline
\end{tabular}

constant pattern of paralysis not present in these cases, is progressive. and lacks distinctive radiological features.

The one example of vertical talus occurring in association with neurofibromatosis is a curiosity not mentioned by McCarroll (1950) in his review of skeletal abnormalities associated with this disease.

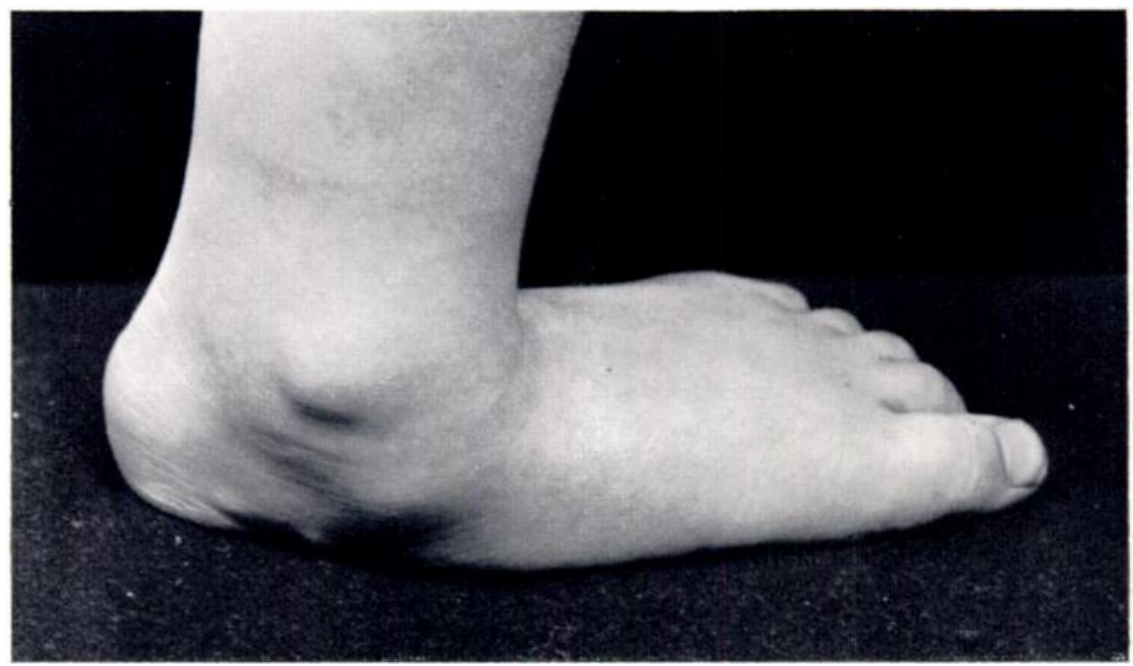

FIG. 8

Severe idiopathic flat foot in a child of four. The heel touches the ground.

\section{DIFFERENTIAL DIAGNOSIS}

Idiopathic flat foot-The deformity on standing may approach in severity that seen in congenital vertical talus, but the whole lower surface of the heel remains on the ground (Fig. 8). At rest the foot does not appear deformed, and the valgus heel, which is not in equinus, can be placed readily in a neutral position. In early childhood the head of the talus can be pushed up from 
the sole and, like the valgus heel, it offers no resistance to passive correction until early adolescence, when inversion at the subtalar joint may become restricted. There may. however, be early limitation of dorsiflexion, detectable only when the heel is held in the neutral position,

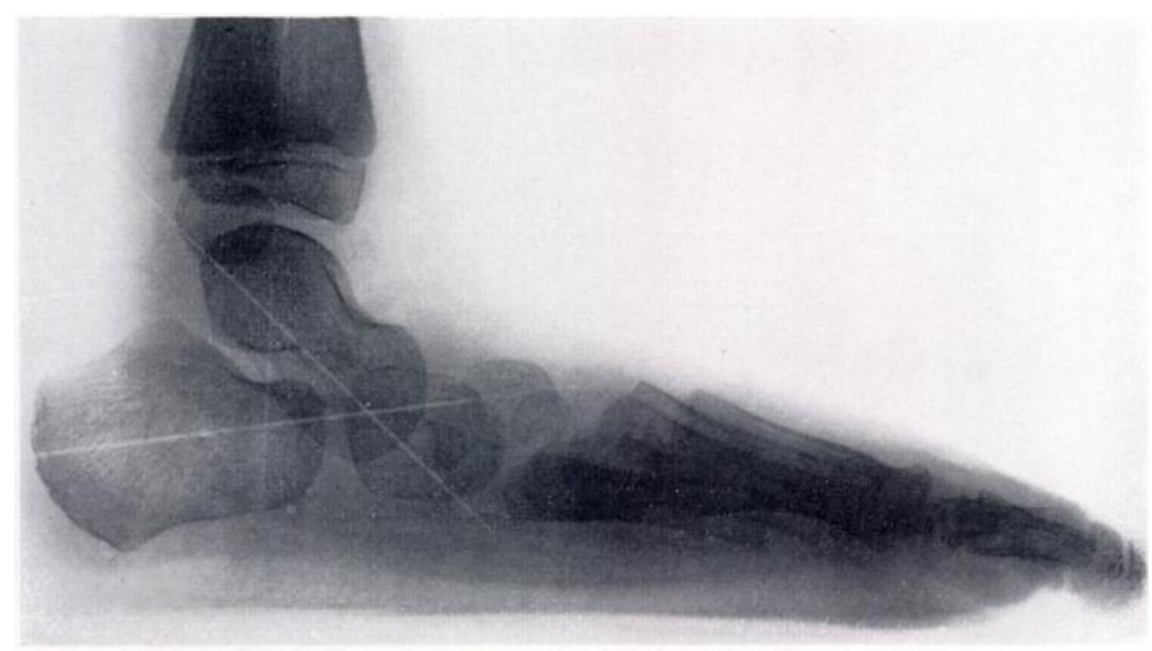

FIG. 9

Severe flat foot with talo-navicular subluxation in a child of five years. The talus inclines towards the perpendicular but there are no other abnormalities in the standing radiograph.

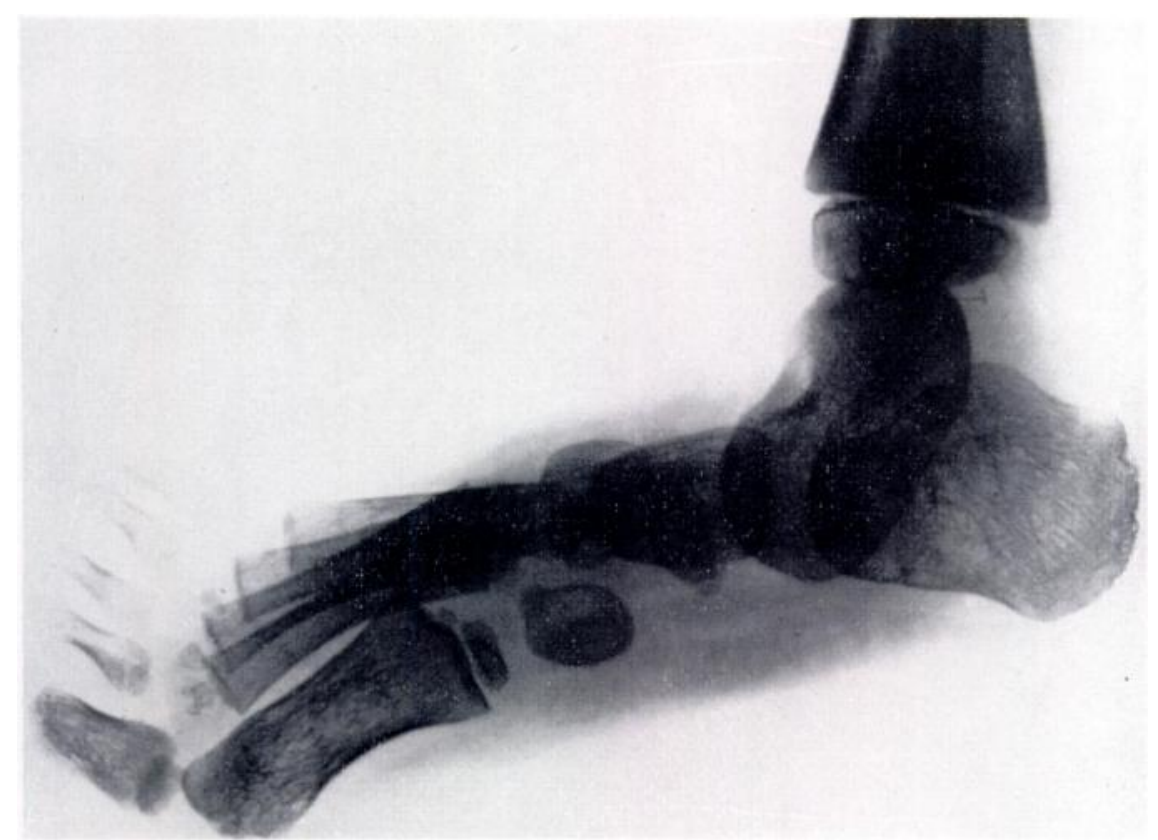

Fig. 10

The same patient as in Figure 9, radiographed on the same day, but with the film badly centred and the patient not bearing weight. Although the talus is vertical there are no other abnormalities.

and caused by shortening of the calcaneal tendon, either primary or secondary (Harris and Beath 1948). The prominence of the head of the talus in a mobile flat foot does not precede weight bearing, whereas the prominence with congenital vertical talus is present at birth, fixed from the start, and no worse on weight bearing. 
Any doubts can be removed by radiography with the patient standing (Jack 1953). In idiopathic flat foot the talus often becomes displaced towards the sole, but there is never complete talo-navicular dislocation, nor does the talus approach the perpendicular. The calcaneum may be horizontal if the posterior calf muscles are short, but it is never in equinus (Fig. 9). Secondary changes in the body of the talus, navicular and calcaneum do not occur.

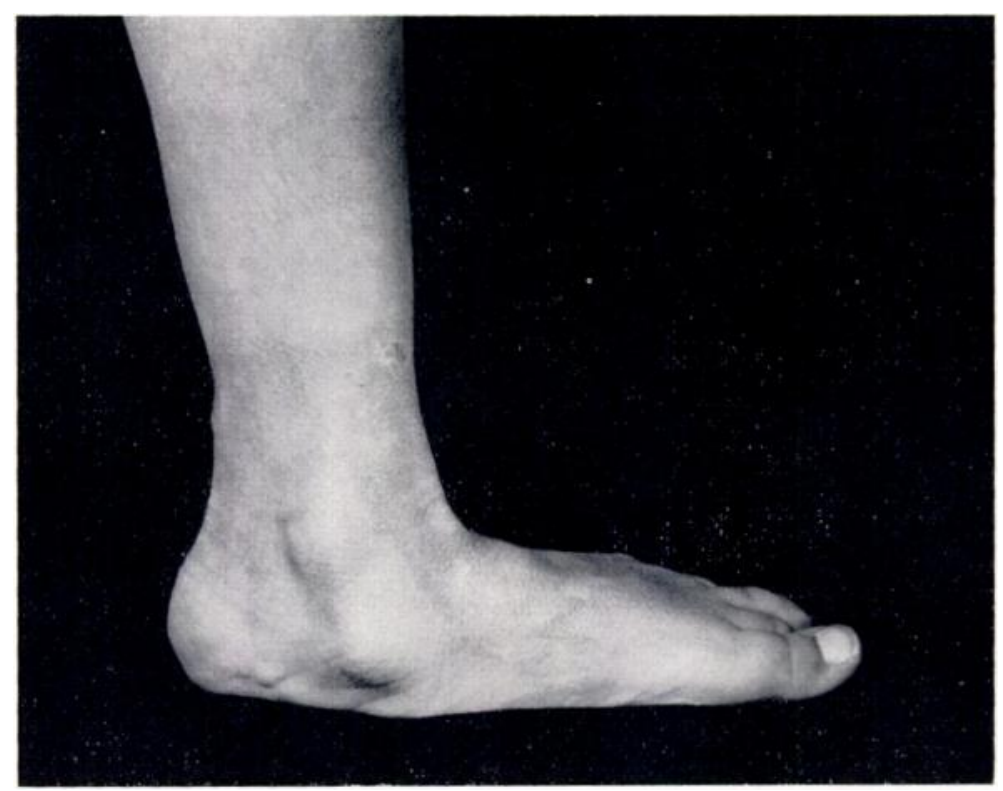

Fig. 11

Severe flat foot in cerebral palsy.

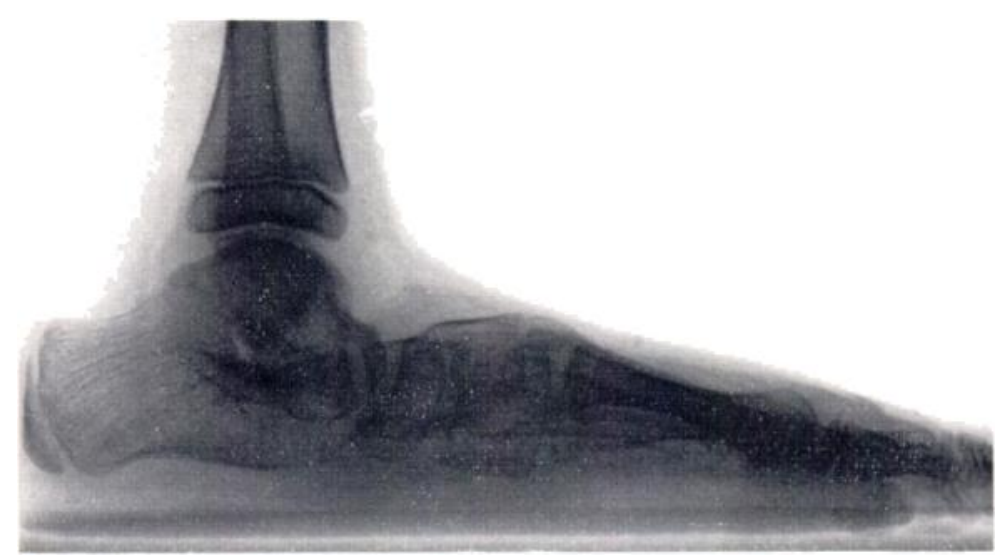

FIci. 12

Radiograph of the foot illustrated in Figure 11. Cerebral palsy in patient aged twelve years. The talus is steep but has neither the characteristic shape nor the other signs shown in Figure 7. The calcaneum is horizontal and the heel touches the ground.

and the forefoot is not dorsiflexed. Mistakes may arise if the radiograph is incorrectly centred, as in Figure 10. On this evidence congenital vertical talus was diagnosed in spite of the lack of changes, such as talo-navicular dislocation. Figure 9 shows the same foot radiographed while the patient was standing.

Paralytic flat foot-This may be caused by anterior poliomyelitis or by spina bifida. Clinical

VOL. $40 \mathrm{~B}$, NO. 1, FEBRUARY 1958 
examination should detect the weakness of the invertors. but even if this is difficult to discover in infancy the mobility of the foot should prevent confusion with congenital vertical talus. When rigidity in eversion develops later it is mainly the shortening of the tendo calcaneus that tethers the calcaneum in valgus, rather than a fixed eversion of the subtalar joint. The radiograph resembles that of severe idiopathic flat foot.

Flat foot in cerebral palsy-Flat foot is a common accompaniment of cerebral palsy and a minor disability in a severely handicapped patient. Nevertheless in very young children with mild cerebral palsy it is an important sign that may be helpful in diagnosis. Confusion with congenital vertical talus may arise because the equino-valgus deformity may become fixed at an early age, but the radiograph again resembles that of idiopathic flat foot. The calcaneum may be horizontal but the heel does not leave the ground (Figs. 11 and 12).

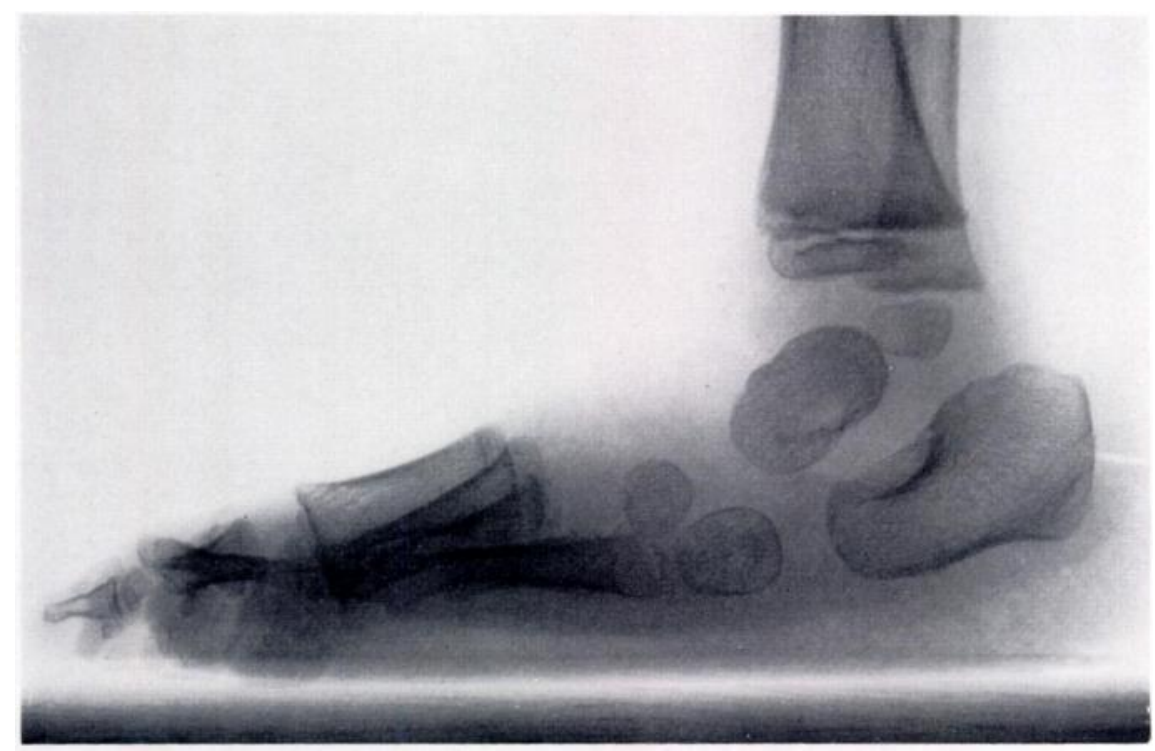

Fig. 13

Uncorrected club foot in patient aged three years. The calcaneum is in equinus. Compare with Figure 5.

Spurious correction of a club foot-When treatment of talipes equino-varus results in spurious correction of equinus at the midtarsal joint the head of the talus becomes prominent and fixed in the sole. In the radiograph the talus and calcaneum are seen to remain in equinus, and the picture may simulate congenital vertical talus. When inversion of the heel has been satisfactorily corrected the resemblance is even greater (Fig. 13). The distinction is likely to be made on the history, and may be supported by the radiograph which shows this iatrogenic vertical talus to differ from the congenital by its broad top and the absence of narrowing of the body.

Talipes calcaneo-valgus-This mistaken diagnosis was made in six infants. It is probable that dorsiflexion of the forefoot distracted the examiner's attention from the equinus deformity of the heel.

\section{TREATMENT}

Fifteen out of twenty-two patients had been treated. We have summarised in Table II the non-operative methods that were used, together with the number of patients treated by each technique; more than one method was used in most patients.

Clearly these methods are too diverse for individual assessment; but they have in common an unsatisfactory outcome, because the deformity was neither corrected nor significantly improved in any instance even when treatment started within the first few weeks of life. 
Operative methods-Tenotomy of the tibialis anterior was performed three times without apparent benefit. Wedge tarsectomy failed to correct the deformity in one child of seven and is to be repeated. Dunn's arthrodesis was carried out twice for pain and deformity in adolescents both aged fourteen, and after a short period of observation the outcome in these is promising.

TABLE II

Methods of Non-operative Treatment Used in Twenty-two Patients (Twenty-eight Feet)

\begin{tabular}{|c|c|}
\hline Denis Browne hobble splint, reversed & 6 \\
\hline Manipulation followed by plaster & 6 \\
\hline Iron and T-strap . & 4 \\
\hline Shoe wedges and stiffening & 5 \\
\hline Corrective night splints & 7 \\
\hline
\end{tabular}

Treatment was not attempted in seven patients, because of mental deficiency or some other dominating disability and the pessimism that followed analysis of our results.

\section{SUMMARY AND CONCLUSIONS}

1. The clinical and radiological features of thirty-two feet with congenital vertical talus are described and subdivided into groups determined by the presence or absence of associated abnormalities.

2. The differential diagnosis of congenital vertical talus from flat foot, talipes calcaneus and uncorrected club foot is discussed.

3. No benefit came from either non-operative treatment or tenotomy of the tibialis anterior.

It gives us pleasure to thank $\mathrm{Mr}$ Denis Browne for his interest and for his permission to review patients under his care at the Hospital for Sick Children, Mr K. I. Nissen for permission to include two of his patients, Mr A. T. Fripp and Mr David Trevor for allowing us to review one patient each and the latter for Figure 6. We are indebted to $\mathrm{Mr} \mathbf{J}$. H. Shelswell for providing us with an early radiograph of one of our cases (Fig. 1). Mr H. Jackson Burrows has given us valuable help in the preparation of this report. We would like to thank Mr Derek Martin, who was responsible for the illustrations, and the Medical Record Department of the Royal National Orthopaedic Hospital.

\section{REFERENCES}

Browne, D. (1956): Personal communication.

HARK, F. W. (1950): Rocker-foot due to Congenital Subluxation of the Talus. Journal of Bone and Joint Surgery, 32-A, 344.

Harris, R. I., and BeAth, T. (1948): Hypermobile Flat-foot with Short Tendo Achillis. Journal of Bone and Joint Surgery, 30-A, 116.

JACK, E. A. (1953): Naviculo-cuneiform Fusion in the Treatment of Flat Foot. Journal of Bone and Joint Surgery, 35-B, 75.

Lamy, L., and Weissman, L. (1939): Congenital Convex Pes Valgus. Journal of Bone and Joint Surgery, $21,79$. MCCARroll, H. R. (1950): Clinical Manifestations of Congenital Neurofibromatosis. Journal of Bone and Joint Surgery, 32-A, 601.

Osmond-Clarke, H. (1956): Congenital Vertical Talus. Journal of Bone and Joint Surgery, 38-B, 334. 